\title{
TAXONOMIC ENUMERATION OF ANGIOSPERM FLORA OF SREENAGAR UPAZILA, MUNSHIGANG, DHAKA, BANGLADESH
}

\author{
ZAKIA MAHMUDAH, MD. MUZAHIDUL ISLAM, \\ TAHMINA HAQUE AND MOHAMMAD ZASHIM UDDIN ${ }^{1}$ \\ Department of Botany, University of Dhaka, Dhaka-1000, Bangladesh
}

\begin{abstract}
The present article focuses the status of angiosperm flora of Sreenagar upazila under Munshiganj district. The study was done from July 2015 to June 2016. A total of 219 plant species of angiosperms was identified belonging to 165 genera and 70 families. Among them 38 species were monocotyledons and 181 plant species were dicotyledons. Herbs were the largest life forms among the angiosperms and contained about 58\% of total plant species occurring in this area. Trees and shrubs occupied 23\% and $12 \%$ respectively. Climbers were $6 \%$ but epiphytes $(1 \%)$ were very negligible in number in the study area. About 51 medicinal plants were recorded from this study. The following species viz. Lasia spinosa, Calamus tenuis, Tinospora crispa, Passiflora foetida and Calotropis procera were recorded only once and hence considered as rare species in Sreenagar upazila. An invasive poisonous plant Parthenium hysterophorus was also found in Sreenagar.
\end{abstract}

Key words: Diversity, Angiosperm flora, Sreenagar, Munshiganj district

\section{Introduction}

Sreenagar is an upazila under Munshiganj district situated on the bank of 'Padma' river. It is a part of Dhaka division, located in between $23^{\circ} 27^{\prime}$ and $23^{\circ} 38^{\prime}$ north latitudes and in between $90^{\circ} 10^{\prime}$ and $90^{\circ} 22^{\prime}$ east longitudes. The total area is 202,98 square kilometer and bounded by Serajdikhan and Nawabganj upazilas on the north, Lohajong and Shibchar upazilas on the south, Serajdikhan and Nawabganj and Dohar upazilas on the west. It consists of 14 union parishads, 102 mouzas and 148 villages. The temperature of this area fluctuates between $13.7{ }^{\circ} \mathrm{C}$ and $37.7{ }^{\circ} \mathrm{C}$ throughout the year. Monthly average relative humidity varies from 54 to $82 \%$ throughout the year. The highest precipitation was found in July. Sreenagar upazila presents diverse types of habitats, e.g. char lands, riparian, homestead, roadside, wetland (Sarker 2012).

Angiosperm flora of Sreenagar upazila has great economic and cultural importance especially in providing food, medicine, fuel and shelter for the local people. It also plays a key role in maintaining the environmental balance and ecosystem stability of the area. Plants of this area have been facing many threats. These are habitat loss and fragmentation, introduction of exotic species, loss of pollinators, over exploitation, pollution and developmental work for (e.g. Padma bridge construction). A good number

\footnotetext{
${ }^{1}$ Corresponding author: zashim01@ gmail.com
} 
of floristic studies on the angiosperm flora of different areas of Bangladesh had already been published. (Khan et al. 1994, Rahman and Hassan 1995, Uddin et al. 1998, Khan and Huq 2001, Uddin et al. 2011, Uddin and Hassan et al. 2004, 2010; Uddin et al. 2013 and Rahman et al. 2013). But there was no study on the angiosperm flora of Sreenagar Upazila. Thus this study was conducted to know the present status of angiosperm flora of Sreenagar Upazila and to find out the threats and stress for the plant community of this area.

\section{Materials and Methods}

Plant samples were collected from the study area in different seasons during July 2015 to June 2016 through six repeated field trips. The area was divided into 14 spots (Sreenagar upazila, Atpara, Tantar, Kukutia, Sholaghar, Rarikhal, Hasara, Kolapara, Shamsiddhi, Bhagyakul, Bhagra, Birtarafor, Baraikhali, Patabhog) for collecting plant samples. The survey covered all habitats and ecosystems of the study site including homestead, road sides, char lands, aquatic bodies and cultivated lands. Voucher specimens processed using standard herbarium techniques (Hyland 1972). The specimens were identified consulting different Floras viz., Hooker 1872-1897, Prain 1903, Uddin and Hassan 2004, Siddiqui et al. (2007c) and Ahmed et al. (2008a, 2008b, 2009b, 2009c, 2009d, 2009e). Specimens available at Dhaka University Salar Khan Herbarium (DUSH) were consulted in identifying the collected plant specimens. The updated nomenclature of the species followed Siddiqui et al. (2007c) and Ahmed et al. (2008a, 2008b, 2009b, 2009c, 2009d, and 2009e). Voucher specimens are deposited at DUSH. The species representation in the families varied from 1 to 16 . In both monocot and dicot angiosperms, Poaceae is the largest family represented by 16 species and Asteraceae and Moraceae is the second largest family represented by 11 species Fig.1 showing the top dominated families. Poaceae, Asteraceae were also reported to be the largest family in a previous study from Bangladesh (Uddin and abiabdullah 2016 and Rahman et al. 2013).

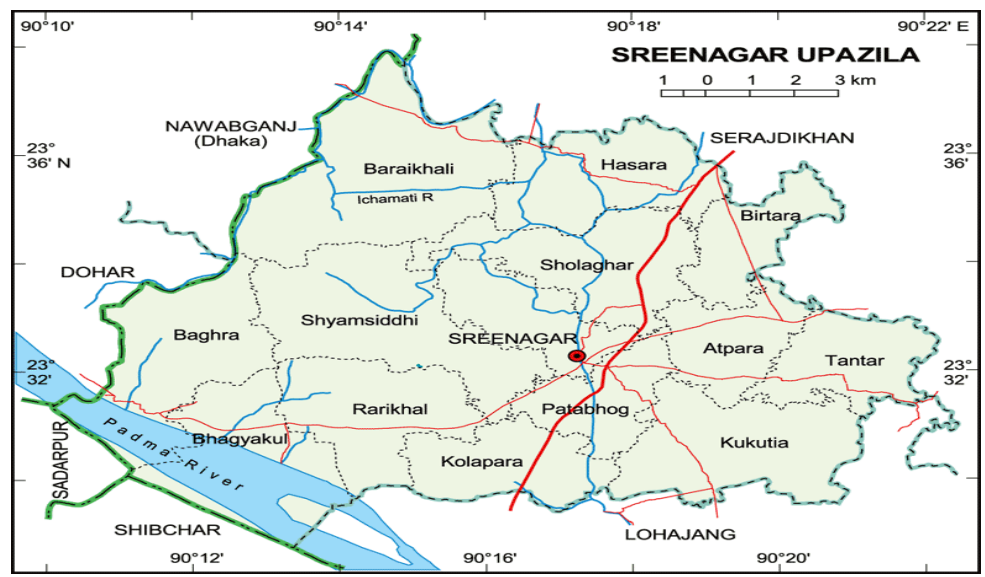

Fig. 1. Map of Sreenagar Upazila. 


\section{Results and Discussion}

A total of 219 angiosperm species was identified under 165 genera and 70 families. Among them Magnoliopsida (dicotyledons) is represented by 181 Species belonging to 129 genera and 58 families, whereas Liliopsida (monocotyledons) by 38 species under 36 genera and 12 families only. For each species local name, scientific name, family, habit and habitat are presented in Table 1. Among the species, 126 are represented by herbs, 26 by shrubs, 54 by trees and 12 by climbers and epiphyte (Fig. 3). Poaceae having 16 species is the largest family in monocotyledon whereas Asteraceae and Moraceae both having 11 species in each family in dicotyledon. Local people use different plants for medicinal purposes. 51 medicinal plants were found from this study. It is about $23 \%$ of the total flora. Roadside vegetation occupied the highest number of identified plant species and was $36 \%$ of total species.

Table1. Plant diversity of Sreenagar upazila.

\begin{tabular}{|c|c|c|c|c|c|}
\hline Scientific Name & Local Name & Family Name & Habit & Habitat & Coll. No. \\
\hline $\begin{array}{l}\text { Abelmoschus esculentus (L.) } \\
\text { Moench }\end{array}$ & Dherosh & Malvaceae & $\mathrm{H}$ & Cultivated & 120 \\
\hline Acacia auriculiformis A. & Akashmoni & Mimosaceae & $\mathrm{T}$ & Road side & 52 \\
\hline Acacia nilotica $\mathrm{L}$. & Babla & Fabaceae & $\mathrm{T}$ & Road side & 62 \\
\hline Acalypha indica $\mathrm{L}$. & Muktajhuri & Euphorbiaceae & $\mathrm{S}$ & Road side & 268 \\
\hline Achyranthes aspera $\mathrm{L}$. & Bilaiachra & Amaranthaceae & $\mathrm{H}$ & Road side & 22 \\
\hline $\begin{array}{l}\text { Adenosma indianum (Lour.) } \\
\text { Merr. }\end{array}$ & Barakesuti & Scrophulariaceae & $\mathrm{H}$ & Char & 76 \\
\hline $\begin{array}{l}\text { Adenostemma lavenia (L.) O. } \\
\text { Kuntze }\end{array}$ & Borokesuti & Asteraceae & $\mathrm{H}$ & Road side & 176 \\
\hline Aegle marmelos (L.) Corr. & Bel & Rutaceae & $\mathrm{T}$ & Homestead & 222 \\
\hline Ageratum conyzoides L. & Fulkuri & Asteraceae & $\mathrm{H}$ & Road side & 7 \\
\hline Albizia lebeck (L.) Benth. & Siris & Fabaceae & $\mathrm{S}$ & Road side & 20 \\
\hline Allium cepa $\mathrm{L}$. & Piaj & Amaryllidaceae & $\mathrm{H}$ & Cultivated & 39 \\
\hline Allium sativum $\mathrm{L}$. & Rosun & Amaryllidaceae & $\mathrm{H}$ & Cultivated & 26 \\
\hline $\begin{array}{l}\text { Alocasia cucullata (Lour.) G. } \\
\text { Don }\end{array}$ & Bishkachu & Araceae & $\mathrm{H}$ & Homestead & 174 \\
\hline Aloe vera (L.) Brum. f. & Gritokumari & Aloeaceae & $\mathrm{H}$ & Homestead & 40 \\
\hline Alstonia scholaris (L.) R. Br. & Chatim & Apocynaceae & $\mathrm{T}$ & Road side & 180 \\
\hline $\begin{array}{l}\text { Alternanthera philoxeroides } \\
\text { (Mart.) Griseb. }\end{array}$ & Helencha & Amaranthaceae & $\mathrm{H}$ & Riparian & 168 \\
\hline $\begin{array}{l}\text { Alternanthera sessilis (L.) R. } \\
\text { Br. ex. Roem. \& Schult. }\end{array}$ & Sachi shak & Amaranthaceae & $\mathrm{H}$ & Char & 1 \\
\hline Amaranthus blitum L. & & Amaranthaceae & $\mathrm{H}$ & Cultivated & 233 \\
\hline Amaranthus spinosus L. & Kanta nutia & Amaranthaceae & $\mathrm{S}$ & Cultivated & 30 \\
\hline Amaranthus tricolor $\mathrm{L}$. & Lal shak & Amaranthaceae & $\mathrm{H}$ & Cultivated & 240 \\
\hline Amaranthus viridis $\mathrm{L}$. & Note shak & Amaranthaceae & $\mathrm{T}$ & Cultivated & 46 \\
\hline Annona reticulata $\mathrm{L}$. & Ataphal & Annonaceae & $\mathrm{T}$ & Homestead & 83 \\
\hline Annona squamosa $\mathrm{L}$. & Sharifa & Annonaceae & $\mathrm{T}$ & Homestead & 279 \\
\hline Areca catech $\mathrm{L}$. & Supari & Arecaceae & $\mathrm{T}$ & Homestead & 152 \\
\hline $\begin{array}{l}\text { Artocarpus heterophyllus } \\
\text { Lamk. }\end{array}$ & Kathal & Moraceae & $\mathrm{T}$ & Homestead & 192 \\
\hline $\begin{array}{l}\text { Artocarpus lakucha Buch.- } \\
\text { Ham. }\end{array}$ & Dewa & Moraceae & $\mathrm{T}$ & Homestead & 163 \\
\hline Averrhoa carambola $\mathrm{L}$. & Kamranga & Oxalidaceae & $\mathrm{H}$ & Homestead & 215 \\
\hline Azadirachta indica A. Juss. & Nim & Meliaceae & $\mathrm{T}$ & Homestead & 64 \\
\hline
\end{tabular}


Contd.

\begin{tabular}{|c|c|c|c|c|c|}
\hline Scientific Name & Local Name & Family Name & Habit & Habitat & Coll. No. \\
\hline $\begin{array}{l}\text { Barringtonia acutangula (L.) } \\
\text { Gaertn. }\end{array}$ & Hijol & Lecythidaceae & $\mathrm{H}$ & Riparian & 290 \\
\hline Basella alba $\mathrm{L}$. & Puishak & Basellaceae & $\mathrm{H}$ & Homestead & 213 \\
\hline Bauhinia variegata $\mathrm{L}$. & Lal kanchon & Caesalpiniaceae & $\mathrm{T}$ & Homestead & 242 \\
\hline Blumea lacera (Burm. f.) DC. & Shialmdra & Asteraceae & $\mathrm{H}$ & Road side & 266 \\
\hline $\begin{array}{l}\text { Boehmeria nivea } \\
\text { (L.)Gaudich. }\end{array}$ & - & Utricaceae & $\mathrm{H}$ & Road side & 87 \\
\hline Bombax ceiba $\mathrm{L}$. & Shimul & Bombacaceae & $\mathrm{H}$ & Road side & 49 \\
\hline Borassus flabellifer $\mathrm{L}$. & Tal & Arecaceae & $\mathrm{T}$ & Road side & 50 \\
\hline Bougainvillea glabra Choisy. & Baganbilas & Nyctaginaceae & $\mathrm{H}$ & Homestead & 288 \\
\hline Cajanus cajan(L.) Millsp. & Arhor dal & Fabaceae & $\mathrm{T}$ & Road side & 194 \\
\hline Calamus tenuis Roxb. & Bet & Arecaceae & $\mathrm{T}$ & Riparian & 73 \\
\hline $\begin{array}{l}\text { Calotropis gigantea (L.) R. } \\
\text { Br. }\end{array}$ & Akondo & Asclepiadaceae & $S$ & Road side & 200 \\
\hline Capsicum frutescens $\mathrm{L}$. & Morich & Solanaceae & $\mathrm{H}$ & Cultivated & 185 \\
\hline $\begin{array}{l}\text { Cardiospermum helicacabum } \\
\text { L. }\end{array}$ & Vat & Sapindaceae & $\mathrm{H}$ & Road side & 61 \\
\hline Carica papaya $\mathrm{L}$. & Pepe & Caricaceae & $\mathrm{T}$ & Homestead & 220 \\
\hline Carissa carandas $\mathrm{L}$. & Koromcha & Apocynaceae & $\mathrm{T}$ & Homestead & 283 \\
\hline Caryota urens $\mathrm{L}$. & Fish tail palm & Arecaceae & $\mathrm{H}$ & Road side & 287 \\
\hline Cassia javanica $\mathrm{L}$. & Java sonalu & Caesalpiniaceae & $\mathrm{T}$ & Road side & 179 \\
\hline $\begin{array}{l}\text { Cassia nodosa Buch.-Ham. ex } \\
\text { Roxb. }\end{array}$ & Bansonalu & Caesalpiniaceae & $\mathrm{H}$ & Road side & 60 \\
\hline Casuarina equisetifolia Forst. & Jhaw & Casuarinaceae & $\mathrm{H}$ & Char & 286 \\
\hline $\begin{array}{l}\text { Catharanthus roseus (L.) G. } \\
\text { Don }\end{array}$ & Noyon tara & Apocynaceae & $\mathrm{H}$ & Homestead & 70 \\
\hline Cayratia trifolia (L.) Domin. & Gowalialata & Vitaceae & $\mathrm{C}$ & Road side & 265 \\
\hline Celosia argentea $\mathrm{L}$. & Morog ful & Amaranthaceae & $\mathrm{H}$ & Homestead & 71 \\
\hline Centella asiatica (L.) Urban. & Thankune & Apiaceae & $\mathrm{H}$ & Homestead & 165 \\
\hline Cestrum nocturnum $\mathrm{L}$. & Hasna hena & Solanaceae & $\mathrm{S}$ & Homestead & 246 \\
\hline $\begin{array}{l}\text { Chamaecrista mimosoides } \\
\text { (L.) Greene. }\end{array}$ & - & Caesalpiniaceae & $\mathrm{H}$ & & 21 \\
\hline Chenopodium album $\mathrm{L}$. & Bothua shak & Chenopodiaceae & $\mathrm{T}$ & Char & 295 \\
\hline Chloris gayana Kunth. & - & Poaceae & $\mathrm{C}$ & & 10 \\
\hline $\begin{array}{l}\text { Chromolaena odorata (L.) } \\
\text { King \& Robinson. }\end{array}$ & Assamlata & Asteraceae & $\mathrm{H}$ & Road side & 19 \\
\hline $\begin{array}{l}\text { Chylocalyx perfoliatus (L.) } \\
\text { Hassk.ex Miq. }\end{array}$ & Kantatok pata & Polygonaceae & $\mathrm{H}$ & Char & 53 \\
\hline Citrus limon (L.) Burm. & Lebu & Rutaceae & $S$ & Homestead & 45 \\
\hline Citrus maxima (Burm.) Merr. & $\begin{array}{l}\text { Batabi } \\
\text { lebu,Jambura }\end{array}$ & Rutaceae & $S$ & Homestead & 150 \\
\hline Cleome gynandra $\mathrm{L}$. & Hurhuria & Capparaceae & $\mathrm{H}$ & Road side & 136 \\
\hline Cleome rutidosperma DC. & & Capparaceae & $\mathrm{T}$ & Riparian & 32 \\
\hline Coccinia grandis (L.) Voigt. & Telakucha & Cucurbitaceae & $\mathrm{C}$ & Road side & 43 \\
\hline Cocos nucifera $\mathrm{L}$. & Narikel & Arecaceae & $\mathrm{T}$ & Homestead & 88 \\
\hline $\begin{array}{l}\text { Codiaeum variegatum (L.) A. } \\
\text { Juss. }\end{array}$ & - & Euphorbiaceae & $\mathrm{H}$ & Homestead & 172 \\
\hline Coix lacryma-jobi $\mathrm{L}$. & Kunch,Tasbi & Poaceae & $\mathrm{H}$ & Rice field & 128 \\
\hline Commelina benghalensis $\mathrm{L}$. & Kanailota & Commelinaceae & $\mathrm{H}$ & Road side & 82 \\
\hline Conyza aegyptiaca (L.) W. Ait. & - & Asteraceae & $\mathrm{H}$ & Road side & 11 \\
\hline Corchorus capsularis & Deshi pat & Tiliaceae & $\mathrm{H}$ & Cultivated & 97 \\
\hline Crateva magna (Lour.) DC. & Bannya,barun & Capparaceae & $\mathrm{T}$ & Riparian & 297 \\
\hline
\end{tabular}


Contd.

\begin{tabular}{|c|c|c|c|c|c|}
\hline Scientific Name & Local Name & Family Name & Habit & Habitat & $\begin{array}{l}\text { Coll. } \\
\text { No. }\end{array}$ \\
\hline Croton bonplandianus Baill. & Bondhone & Euphorbiaceae & $\mathrm{H}$ & Road side & 8 \\
\hline Cucumis sativus L. & Shasa,Khira & Cucurbitaceae & $\mathrm{C}$ & Cultivated & 126 \\
\hline Cucurbita maxima Duch.ex Lamk. & Mistialu & Cucurbitaceae & $\mathrm{C}$ & Cultivated & 65 \\
\hline Cucurbita moschata Duch. Ex Poir. & Safra kumra & Cucurbitaceae & $\mathrm{H}$ & Cultivated & 143 \\
\hline Cuscuta reflexa Roxb. & Swarnalata & Cuscutaceae & Epiphyte & $\begin{array}{l}\text { Epiphyte, } \\
\text { Roadside }\end{array}$ & 284 \\
\hline Cyclea barbata Miers. & Patalpur & Menispermaceae & $\mathrm{H}$ & Road side & 5 \\
\hline Cynodon dactylon $L$. & Durba & Poaceae & $\mathrm{H}$ & open field & 79 \\
\hline Cyperus iria L. & - & Cyperaceae & $\mathrm{H}$ & Cultivated land & 167 \\
\hline Cyperus rotundus & Mutha gash & Cyperaceae & $\mathrm{H}$ & Cultivated land & 13 \\
\hline $\begin{array}{l}\text { Dactyloctenium } \\
\text { aegyptium(L.) P.Beauv. }\end{array}$ & Makra & Poaceae & $\mathrm{H}$ & Road side & 80 \\
\hline Dalbergia sisso Roxb. & Shisu & Fabaceae & $\mathrm{S}$ & Road side & 48 \\
\hline Datura metel L. & Dhutra & Solanaceae & $\mathrm{H}$ & Road side & 267 \\
\hline $\begin{array}{l}\text { Dendrocalamus strictus } \\
\text { Roxb. Nees. }\end{array}$ & Karail & Poaceae & $\mathrm{H}$ & Rice field & 89 \\
\hline Digitaria bicornis (Lamk.) & - & Poaceae & $\mathrm{H}$ & Road side & 14 \\
\hline $\begin{array}{l}\text { Roem. \& Schult. ex Loud. } \\
\text { Digitaria stricta Roth ex } \\
\text { Roem. \& Schult. }\end{array}$ & Ghash & Poaceae & $\mathrm{H}$ & Road side & 18 \\
\hline $\begin{array}{l}\text { Digitaria ternata (A. Rich.) } \\
\text { Stapf ex Dyer. }\end{array}$ & Ghash & Poaceae & $\mathrm{H}$ & Road side & 91 \\
\hline Dillenia indica $\mathrm{L}$. & Chalta & Dilleniaceae & $\mathrm{T}$ & Homestead & 93 \\
\hline Dioscorea alata $\mathrm{L}$. & Chupri alu & Dioscoriaceae & $\mathrm{T}$ & Homestead & 92 \\
\hline $\begin{array}{l}\text { Diospyros discolor } \\
\text { Willd.nom illeg.Verheij \& }\end{array}$ & Bilatigab & Ebenaceae & $\mathrm{T}$ & Homestead & 119 \\
\hline $\begin{array}{l}\text { Coronel. } \\
\text { Diospyros malabarica (Desr. } \\
\text { Kostel. }\end{array}$ & & Ebenaceae & $\mathrm{T}$ & Homestead & 42 \\
\hline Duranta repens L. & Duranta & Verbenaceae & $\mathrm{H}$ & Homestead & 271 \\
\hline $\begin{array}{l}\text { Echinochloa colonum (L.) } \\
\text { Link. }\end{array}$ & Buno Dhan & Poaceae & $\mathrm{H}$ & Road side & 158 \\
\hline $\begin{array}{l}\text { Eichhornia crassipes (Mart.) } \\
\text { Solms. }\end{array}$ & Kochuripana & Pontederiaceae & $\mathrm{H}$ & Aquatic & 161 \\
\hline Elaeocarpus tectorius (Lour.) Poir. & Jolpai & Elaeocarpaceae & $\mathrm{H}$ & Homestead & 182 \\
\hline $\begin{array}{l}\text { Eucalyptus camaldulensis } \\
\text { Dehnhardt. }\end{array}$ & Eucalyptus & Myrtaceae & $\mathrm{T}$ & Road side & 107 \\
\hline Euphorbia hirta L. & Dudhia & Euphorbiaceae & $\mathrm{H}$ & Road side & 37 \\
\hline Euphorbia thymifolia L. & Dudhiya & Euphorbiaceae & $\mathrm{H}$ & Road side & 260 \\
\hline Ficus benghalensis L. & Bot & Moraceae & $\mathrm{T}$ & Road side & 124 \\
\hline Ficus heterophylla & Bot & Moraceae & $\mathrm{T}$ & Road side & 162 \\
\hline Ficus hispida L. f. & Kak dumur & Moraceae & $\mathrm{T}$ & Road side & 177 \\
\hline Ficus racemosa $\mathrm{L}$. & Dumur & Moraceae & $\mathrm{T}$ & Road side & 35 \\
\hline Ficus religiosa L. & Ashok & Moraceae & $\mathrm{T}$ & Road side & 101 \\
\hline Ficus rumphii Blume. & Gaiaswathwa & Moraceae & $\mathrm{T}$ & Road side & 59 \\
\hline Fioria vitifolia (L.) Mattei. & - & Malvaceae & $\mathrm{H}$ & Road side & 110 \\
\hline Glinus oppositifolius (L.) A. DC & Gimashak & Molluginaceae & $\mathrm{H}$ & Char & 296 \\
\hline $\begin{array}{l}\text { Glycosmis pentaphylla } \\
\text { (Retz.) A. DC. }\end{array}$ & Matkila,Datmajoni & Rutaceae & $\mathrm{S}$ & Road side & 131 \\
\hline Heliotropium indicum L. & Hatishur & Boraginaceae & $\mathrm{H}$ & Cultivated & 78 \\
\hline Hibiscus fragrans Roxb. & Joba & Malvaceae & S & Homestead & 99 \\
\hline Hibiscus rosa-sinensis L. & Joba & Malvaceae & S & Homestead & 285 \\
\hline $\begin{array}{l}\text { Hibiscus schizopetalus } \\
\text { (Mast.) Hook. f. }\end{array}$ & Jhumko Joba & Malvaceae & S & Homestead & 291 \\
\hline
\end{tabular}


Contd.

\begin{tabular}{|c|c|c|c|c|c|}
\hline Scientific Name & Local Name & Family Name & Habit & Habitat & Coll. No. \\
\hline Hibiscus surattensis L. & Joba & Malvaceae & $\mathrm{S}$ & Road side & 210 \\
\hline $\begin{array}{l}\text { Hydrocotyle sibthorpioides } \\
\text { Lamk. }\end{array}$ & Unknown & Apiaceae & $\mathrm{H}$ & Aquatic & 121 \\
\hline $\begin{array}{l}\text { Hygrophila schulli (Buch.- } \\
\text { Ham.) M. R. \& S. N. Almeida. }\end{array}$ & Talmakhna & Acanthaceae & $\mathrm{H}$ & Aquatic & 171 \\
\hline Indigofera tinctoria $\mathrm{L}$. & Nil & Fabaceae & S & Cultivated & 90 \\
\hline Ipomea indica (Burm.f.) Merr. & Kolmi & Convolvulaceae & $\mathrm{H}$ & Aquatic & 135 \\
\hline Ipomoea aquatica Forssk. & Kolmi & Convolvulaceae & $\mathrm{H}$ & Aquatic & 145 \\
\hline Ipomoea batatas (L.) Lamk. & Mistialu & Convolvulaceae & $\mathrm{H}$ & Cultivated & 303 \\
\hline $\begin{array}{l}\text { Ipomoea fistulosa Mart. ex } \\
\text { Choisy. }\end{array}$ & - & Convolvulaceae & $\mathrm{H}$ & Aquatic & 311 \\
\hline Ixora coccineaL. & Rongon & Rubiaceae & $\mathrm{S}$ & Homestead & 300 \\
\hline Justicia gendarussa Burm. f. & Jogotmordon & Acanthaceae & $\mathrm{H}$ & Homestead & 103 \\
\hline Kyllinga brevifolia Rottb. & & Cyperaceae & $\mathrm{T}$ & Road side & 146 \\
\hline $\begin{array}{l}\text { Lagenaria siceraria (Molina) } \\
\text { Standl. }\end{array}$ & Lau & Cucurbitaceae & $\mathrm{C}$ & Cultivated & 209 \\
\hline Lasia spinosa (L.) Thw. & Kanta kachu & Araceae & $\mathrm{H}$ & Riparian & 151 \\
\hline Lawsonia inermis L. & Mehedi & Lythraceae & $\mathrm{S}$ & Homestead & 140 \\
\hline $\begin{array}{l}\text { Lepidagathis incurva Buch- } \\
\text { Ham. ex D. Don. }\end{array}$ & - & Acanthaceae & $\mathrm{H}$ & Homestead & 142 \\
\hline $\begin{array}{l}\text { Leucas cephalotes (Roth.) } \\
\text { Spreng. }\end{array}$ & Bara halkus & Lamiaceae & $\mathrm{H}$ & Homestead & 276 \\
\hline Limonia acidissima $\mathrm{L}$. & Kotbel & Rutaceae & $\mathrm{T}$ & Homestead & 123 \\
\hline $\begin{array}{l}\text { Lippia alba (Mill.) Briton et } \\
\text { Wilson. }\end{array}$ & Lippia & Verbenaceae & $\mathrm{H}$ & Char & 211 \\
\hline Litchi chinensis Sonn. & Lichu & Sapindaceae & $\mathrm{T}$ & Homestead & 138 \\
\hline Ludwigia adscendens (L.) Hara. & Mulsi & Onagraceae & $\mathrm{h}$ & Aquatic & 72 \\
\hline $\begin{array}{l}\text { Ludwigia hyssopifolia (G. } \\
\text { Don) Exell. }\end{array}$ & - & Onagraceae & $\mathrm{H}$ & Aquatic & 51 \\
\hline Ludwigia perennis L. & - & Onagraceae & $\mathrm{H}$ & Aquatic & 313 \\
\hline Malvaviscus arboreus Cav. & Joba & Malvaceae & $\mathrm{S}$ & Homestead & 195 \\
\hline Mangifera indica $\mathrm{L}$. & Aam & Anacardiaceae & $\mathrm{T}$ & Homestead & 187 \\
\hline Melia azedarach $\mathrm{L}$. & Goranim & Meliaceae & $\mathrm{T}$ & Road side & 69 \\
\hline $\begin{array}{l}\text { Melocanna baccifera } \\
\text { (Roxb.)Kurz. }\end{array}$ & Muli bash & Poaceae & $\mathrm{H}$ & Cultivated & 147 \\
\hline $\begin{array}{l}\text { Mikania cordata (Burm.f.) } \\
\text { Robinson. }\end{array}$ & Asamlata,Tarulata & Asteraceae & $\mathrm{C}$ & Road side & 148 \\
\hline Mirabilis jalapa $\mathrm{L}$. & Shondha moni & Nyctaginaceae & $\mathrm{S}$ & Homestead & 157 \\
\hline Mollugo pentaphylla $\mathrm{L}$. & Khetp & Molluginaceae & $\mathrm{H}$ & Char & 56 \\
\hline Momordica charantia $\mathrm{L}$. & Uchchhe & Cucurbitaceae & $\mathrm{C}$ & homestead & 235 \\
\hline Monochoria hastata (L.) Solms. & & Pontederiaceae & $\mathrm{H}$ & Aquatic & 17 \\
\hline Moring a oliefera Lamk. & Sajina & Moringaceae & $\mathrm{T}$ & Homestead & 57 \\
\hline Morus macroura Miq. & Tute & Moraceae & $\mathrm{T}$ & Road side & 183 \\
\hline Murraya paniculata (L.) Jack. & Kamini & Rutaceae & $\mathrm{S}$ & Homestead & 141 \\
\hline Musa paradisiaca $\mathrm{L}$. & Kola & Musaceae & $\mathrm{H}$ & Homestead & 203 \\
\hline $\begin{array}{l}\text { Mussaenda erythrophylla } \\
\text { Schum. \& Thonn. }\end{array}$ & Macchenda & Rubiaceae & S & Homestead & 129 \\
\hline $\begin{array}{l}\text { Neolamarckia cadamba } \\
\text { (Roxb.) Bosser. }\end{array}$ & Kokom & Rubiaceae & $\mathrm{T}$ & Road side & 125 \\
\hline Nymphaea nouchali Burm. f. & Nil shapla & Nym & $\mathrm{H}$ & Aquatic & 149 \\
\hline Nymphaea pubescens Willd & Shapla & Nymphaeaceae & $\mathrm{H}$ & Aquatic & 160 \\
\hline Ocimum basilicum L. & Babui tulsi & Lamiaceae & $\mathrm{S}$ & Homestead & 38 \\
\hline Ocimum gratissimum $\mathrm{L}$. & Pantulsi & Lamiaceae & $\mathrm{T}$ & Homestead & 75 \\
\hline Oryza sativa $\mathrm{L}$. & Dhan & Poaceae & $\mathrm{H}$ & cultivated & 159 \\
\hline Oxalis corniculata L. & Amrul & Oxalidaceae & $\mathrm{T}$ & open field & 241 \\
\hline
\end{tabular}


Taxonomic enumeration

Contd.

\begin{tabular}{|c|c|c|c|c|c|}
\hline Scientific Name & Local Name & Family Name & Habit & Habitat & Coll. No. \\
\hline Oxalis corymbosa DC. & - & Oxalidaceae & $\mathrm{H}$ & Char & 47 \\
\hline Panicum milliaceum $\mathrm{L}$. & Cheena chaul & Poaceae & $\mathrm{H}$ & Rice field & 164 \\
\hline Parthenium hysterophorus & - & Asteraceae & $\mathrm{H}$ & Char & 166 \\
\hline Paspalum distichum $\mathrm{L}$. & - & Poaceae & $\mathrm{H}$ & Cultivated & 170 \\
\hline Passiflora foetida $\mathrm{L}$. & Passiflora & Passifloraceae & $\mathrm{H}$ & Road side & 184 \\
\hline Pedilanthus tithymaloides Poit. & Rangchita & Euphorbiaceae & $\mathrm{H}$ & Homestead & 199 \\
\hline $\begin{array}{l}\text { Peperomia pellucida (L.) H. B. } \\
\text { \& K. }\end{array}$ & Luchipata & Piperaceae & $\mathrm{H}$ & Road side & 95 \\
\hline $\begin{array}{l}\text { Persicaria glabra } \\
\text { (Willd.)Gomez de la Maza. }\end{array}$ & Lal kukri & Polygonaceae & $\mathrm{H}$ & Riparian & 36 \\
\hline $\begin{array}{l}\text { Persicaria hydropiper (L.) } \\
\text { Spach. }\end{array}$ & Panimorich & Polygonaceae & $\mathrm{H}$ & Char & 28 \\
\hline $\begin{array}{l}\text { Persicaria lanata (Roxb.) } \\
\text { Hassan. }\end{array}$ & $\begin{array}{l}\text { Shet } \\
\text { panimorich }\end{array}$ & Polygonaceae & $\mathrm{H}$ & Riparian & 29 \\
\hline $\begin{array}{l}\text { Persicaria lapathifolia (L.) S. } \\
\text { F. Gray. }\end{array}$ & Panimorich & Polygonaceae & $\mathrm{H}$ & Aquatic & 231 \\
\hline Phoenix sylvestris Roxb. & Khejur & Arecaceae & $\mathrm{T}$ & Road side & 186 \\
\hline Phyla nodiflora (L.) Greene. & Bhuiokra & Verbenaceae & $\mathrm{H}$ & Road side & 308 \\
\hline Phyllanthus emblica L. & Amloki & Phyllanthaceae & $\mathrm{H}$ & Cultivated & 307 \\
\hline Phyllanthus niruri L. & Bhuiamla & Euphorbiaceae & $\mathrm{S}$ & Road side & 81 \\
\hline Phyllunthus reticulatus Poir. & Chitki & Phyllanthaceae & $\mathrm{T}$ & open field & 278 \\
\hline Physalis angulata $\mathrm{L}$. & Fotka & Solanaceae & $\mathrm{H}$ & Road side & 3 \\
\hline Physalis minima L. & Fotka & Solanaceae & $\mathrm{H}$ & Road side & 41 \\
\hline Piper sylvaticum Roxb. & Pan & Piperaceae & $\mathrm{H}$ & Homestead & 44 \\
\hline Pistia stratiotes L. & Topapana & Araceae & $\mathrm{H}$ & Aquatic & 178 \\
\hline Polyalthia longifolia (Sonn.) Thw. & Debdaru & Annonaceae & $\mathrm{T}$ & Road side & 86 \\
\hline Porana paniculata Roxb. & - & Convolvulaceae & $\mathrm{H}$ & Road side & 312 \\
\hline Portulaca grandiflora Hook. & Time ful & Portulacaceae & $\mathrm{H}$ & Homestead & 181 \\
\hline Portulaca oleracea L. & Nunta shak & Portulacaceae & $\mathrm{H}$ & Char & 156 \\
\hline Psidium guajava $\mathrm{L}$. & Peara & Myrtaceae & $\mathrm{S}$ & Homestead & 139 \\
\hline Punica granatum $\mathrm{L}$. & Dalim & Punicaceae & $\mathrm{S}$ & Homestead & 239 \\
\hline Raphanus sativus L. & Mula & Brassicaceae & $\mathrm{H}$ & Homestead & 202 \\
\hline Ricinus communis $\mathrm{L}$. & Bherenda & Euphorbiaceae & $\mathrm{T}$ & Road side & 24 \\
\hline Ruellia tuberosa $\mathrm{L}$. & Chatpoty & Acanthaceae & $\mathrm{H}$ & Road side & 94 \\
\hline Saccharum officinarum $\mathrm{L}$. & Akh & Poaceae & $\mathrm{H}$ & $\begin{array}{l}\text { Cultivated } \\
\text { land }\end{array}$ & 223 \\
\hline Saccharum spontaneum $\mathrm{L}$. & Kash & Poaceae & $\mathrm{H}$ & Riparian & 133 \\
\hline Scoparia dulcis L. & Bondhone & Scrophulariaceae & $\mathrm{H}$ & Road side & 299 \\
\hline $\begin{array}{l}\text { Senna hirsuta (L.) Irwin \& } \\
\text { Barneby. }\end{array}$ & - & Caesalpiniaceae & $\mathrm{H}$ & Road side & 106 \\
\hline $\begin{array}{l}\text { Senna obtusifolia (L.) Irwin \& } \\
\text { Barneby. }\end{array}$ & - & Caesalpiniaceae & $\mathrm{H}$ & Road side & 304 \\
\hline Senna occidentalis Roxb. & Bora chalkesunda & Caesalpiniaceae & $\mathrm{H}$ & Road sic & 113 \\
\hline Senna tora (L.) Roxb. & Kalkasam & Caesalpiniaceae & $\mathrm{T}$ & Homestead & 98 \\
\hline Sesamum indicum $\mathrm{L}$. & Til & Pedaliceae & $\mathrm{C}$ & $\begin{array}{l}\text { Cultivated } \\
\text { land }\end{array}$ & 257 \\
\hline Sesbania bispinosa & Dhaincha & Fabaceae & $\mathrm{H}$ & $\begin{array}{l}\text { Cultivated } \\
\text { land }\end{array}$ & 169 \\
\hline Setaria barbata (Lamk.) Kunth. & - & Poacea & $\mathrm{H}$ & Cultivated & 9 \\
\hline Sida acuta Burm. & Kureta & Malvaceae & $\mathrm{H}$ & Road side & 2 \\
\hline Solanum americanum Mill. & Titbegun & Solanaceae & $\mathrm{H}$ & Road side & 293 \\
\hline Solanum melongena L. & Begun & Solanaceae & $\mathrm{H}$ & Cultivated & 188 \\
\hline
\end{tabular}


Contd.

\begin{tabular}{|c|c|c|c|c|c|}
\hline Scientific Name & Local Name & Family Name & Habit & Habitat & Coll. No. \\
\hline Solanum nigrum $\mathrm{L}$. & Titbegun & Solanaceae & $\mathrm{H}$ & Road side & 114 \\
\hline Solanum tuberosum & Alu & Solanaceae & $\mathrm{H}$ & Cultivated & 189 \\
\hline Spilanthes calva DC. & Surja kannya & Asteraceae & $\mathrm{H}$ & Char & 6 \\
\hline Streblus asper Lour. & Sheora & Moraceae & $\mathrm{T}$ & Road side & 175 \\
\hline Swietenia mahagoni Jacq. & Mehogoni & Meliaceae & $\mathrm{T}$ & Homestead & 153 \\
\hline $\begin{array}{l}\text { Synedrella nodiflora (L.) } \\
\text { Gaertn. }\end{array}$ & - & Asteraceae & $\mathrm{H}$ & open field & 34 \\
\hline Syzygium cumini (L.) Skeels & Jam & Myrtaceae & $\mathrm{T}$ & Homestead & 55 \\
\hline Syzygium samarangense & Jamrul & Myrtaceae & $\mathrm{T}$ & Homestead & 207 \\
\hline $\begin{array}{l}\text { Tabernaemontana divaricata } \\
\text { (L.) R. Br. ex Roem. \& Schult. }\end{array}$ & Togor & Apocynaceae & $S$ & Homestead & 298 \\
\hline Tagetes erecta $\mathrm{L}$. & Gadha & Asteraceae & $\mathrm{H}$ & homestead & 193 \\
\hline Tamarindus indica $\mathrm{L}$. & Tentul & Caesalpiniaceae & $\mathrm{H}$ & Road side & 132 \\
\hline Tectona grandis L. f. & Segun & Dipterocarpaceae & $\mathrm{T}$ & Homestead & 54 \\
\hline $\begin{array}{l}\text { Terminalia arjuna (Roxb. ex } \\
\text { DC.)Wight \& Arn. }\end{array}$ & Arjun & Combretaceae & $\mathrm{H}$ & Road side & 250 \\
\hline $\begin{array}{l}\text { Thevetia peruviana (Pers.) K. } \\
\text { Schum. }\end{array}$ & Halde karabi & Apocynaceae & S & Homestead & 281 \\
\hline $\begin{array}{l}\text { Tinospora crispa (L.) Hook. f. } \\
\& \text { Thoms. }\end{array}$ & Gulancha & Menispermaceae & $\mathrm{C}$ & Homestead & 196 \\
\hline Trema orientalis (L.) Blume. & - & Cannabaceae & $\mathrm{H}$ & Road side & 204 \\
\hline Triticum aestivum $\mathrm{L}$. & Gom & Poaceae & $\mathrm{H}$ & Cultivated & 197 \\
\hline $\begin{array}{l}\text { Typhonium trilobatum (L.) } \\
\text { Schott. }\end{array}$ & Ghet kachu & Araceae & $\mathrm{H}$ & Homestead & 208 \\
\hline Urena lobata $\mathrm{L}$. & Ban okra & Malvaceae & $\mathrm{H}$ & Road side & 4 \\
\hline $\begin{array}{l}\text { Vigna adenantha (Meyer) } \\
\text { Marechal et al. }\end{array}$ & Bonborboti & Fabaceae & $\mathrm{C}$ & Cultivated & 155 \\
\hline Vigna unguiculata (L.) Walp. & Borboti & Fabaceae & $\mathrm{H}$ & Road side & 191 \\
\hline $\begin{array}{l}\text { Wedelia trilobata (L.) A. S. } \\
\text { Hitchc. }\end{array}$ & - & Asteraceae & $\mathrm{H}$ & Road side & 31 \\
\hline $\begin{array}{l}\text { Xanthium indicum Koen ex } \\
\text { Roxb. }\end{array}$ & Gagra & Asteraceae & $\mathrm{C}$ & Road side & 27 \\
\hline Zea mays $\mathrm{L}$. & Vutta & Poaceae & $\mathrm{H}$ & cultivated & 198 \\
\hline Ziziphus oenoplia (L.) Mill. & Boroi & Rhamnaceae & $\mathrm{T}$ & Homestead & 104 \\
\hline
\end{tabular}

( $\mathrm{T}=$ tree, $\mathrm{S}=$ shrub, $\mathrm{H}=$ herb, $\mathrm{C}=$ climber)

In the study area, Barringtonia acutangula (Hijol), Crateva magna, Persicaria lanata (Panimorich), Saccharum spontaneum are commonly found in bank of rivers and canals. Paddy (Oryza sativa), potato (Solanum tuberosum), wheat (Triticum aestivum), sweet pumpkin (Cucurbita pepo) and maize (Zea mays) are the main cultivated crops which are exposed during dry season. In the rainy season the land is mainly used for cultivation of dhaincha (Sesbania bispinosa) and jute (Corchorus capsularis). 


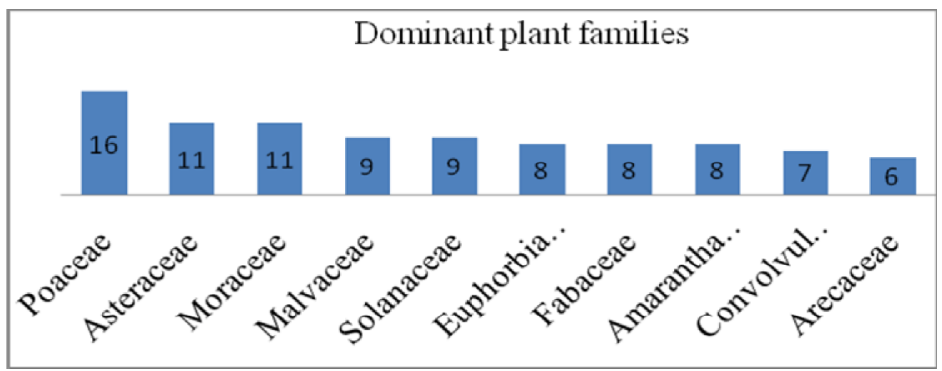

Fig. 2. Top ten dominant families along with the number of species.

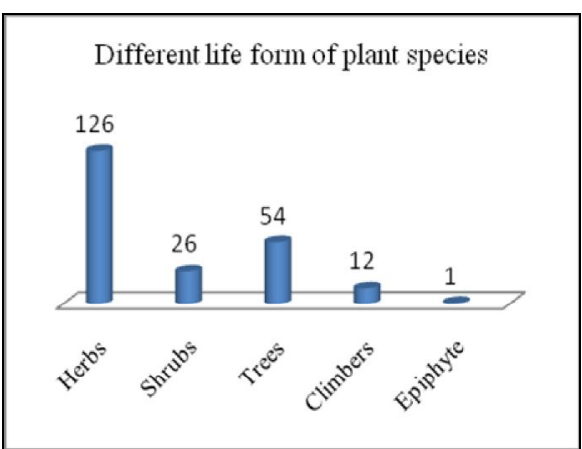

Fig. 3. Different life forms of plant species in Sreenagar Upazila.

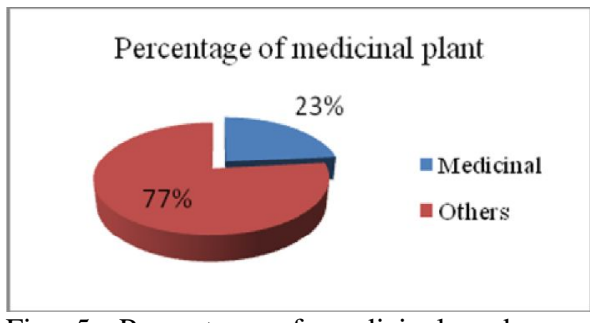

Fig. 5. Percentage of medicinal and non medicinal plant species.

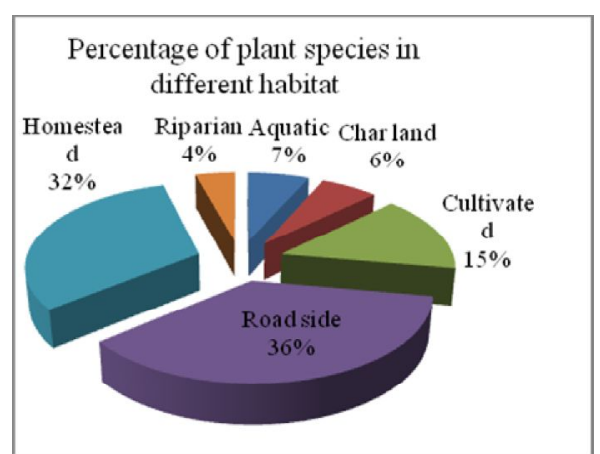

Fig. 4. Percentage of plant species in different habitats in Sreenagar upazila.

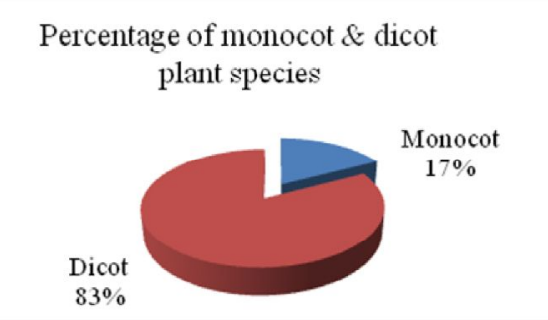

Fig. 6. Percentage of monocot and dicot plant species

The remarkable wild species of the study area are Persicaria lanata, Lippia alba, Streblus asper, Synedrella nodiflora, Trema orientalis, Typhonium trilobatum, Xanthium indicum etc. Alternanthera sessilis, Chenopodium album, Glinus oppositifolius, Phyla nodiflora, Portulaca oleracea, Synedrella nodiflora, Oxalis corniculata are commonly found to form the vegetation in char land. Some common aquatic angiosperms are Eichhornia crassipes, Enhydra fluctuans, Ipomoea aquatica, Ludwigia adscendens, Ludwigia hyssopifolia, Nymphaea nouchali and Pistia stratiotes. 
Crateva magna (Bannya,barun), Barringtonia acutangula (Hijol), Alstonia scholaris, Azadirachta indica, Persicaria lanata, Lippia alba, Phyllunthus reticulatus, Ruellia tuberosa, Sida acuta are more available in the study area.

Calamus tenuis, Coix lacryma-jobi, Lasia spinosa, Tinospora crispa and Passiflora foetida are rarely found in study area. The number of these species is decreasing day by day. Tinospora crispa is a threatened species (Khan et al. 2001) which is a popular medicinal plant used by the local people.

Most common exotic plants of the study area were Acacia auriculiformis (Akashmoni), Acacia nilotica (Babla) and Eucalyptus camaldulensii. Parthenium hysterophorus was recorded as the poisonous exotic plant from sreenagar. The invasive alien species at aquatic habitats namely Eichhornia crassipes, Alternanthera philoxeroides, Ipomoea aquatica and Pistia stratiotes were found to prohibit the growth and dispersal of other aquatic species of the study area destroying the aquatic vegetation. During the field works some threats like destruction of natural habitats by the local people, lack of awareness, unsustainable collection, and river degradation were identified as active in the study area. The wetlands in the study area are brought under cultivating process during the dry season. As a result the aquatic vegetation of these wetlands seems to be diminished. Uses of excessive amount of fertilizers, insecticides, pesticides as well as herbicides are the other reasons for diminishing the natural aquatic vegetation of the study area. Several discussions with local people have been conducted on the conservation issues of angiosperm flora. A number of suggestions came out from such discussion are presented below: (A) Native plants should be selected for plantation. (B) Conservation of the threatened plant species by in-situ methods should be undertaken. The local people should be involved in this activity. (C) Awareness among the local people should be created. (D) Nursery should be developed for the propagation of threatened plants. (E) Government and local NGOs can act jointly for the conservation of angiosperm flora.

\section{Acknowledgements}

The authors are thankful to the Ministry of Science and Technology, Govt. of the People's Republic of Bangladesh for their funding. They are also grateful to the local people of Sreenagar Upazila, Munshiganj.

\section{References}

Ahmed, Z.U., Z.N.T. Begum, M.A. Hassan, M. Khondker, S.M.H. Kabir, M. Ahmad, A.T.A. Ahmed, A.K.A. Rahman and E.U. Haque (Eds) 2008a. Encyclopedia of Flora and Fauna of Bangladesh, Vol. 6. Angiosperms: Dicotyledons (Acanthaceae - Asteraceae). Asiatic Society of Bangladesh, Dhaka, pp. 1-408.

Ahmed, Z.U., M.A. Hassan, Z.N.T. Begum, M. Khondker, S.M.H. Kabir, M. Ahmad, A.T.A. Ahmed, A.K.A. Rahman and E.U. Haque, (Eds)2008b. Encyclopedia of Flora and Fauna 
of Bangladesh, Vol. 12. Angiosperms:Monocotyledons (Orchidaceae - Zingiberaceae). Asiatic Society of Bangladesh, Dhaka, pp. 1-552.

Ahmed, Z.U., Z.N.T. Begum, M.A., Hassan, M. Khondker, S.M.H. Kabir, M. Ahmad, A.T.A. Ahmed, A.K.A. Rahman and E.U. Haque (Eds) 2009b. Encyclopedia of Flora and Fauna of Bangladesh Vol. 7. Angiosperms: Dicotyledons (Balsaminaceae - Euphorbiaceae). Asiatic Society of Bangladesh, Dhaka, pp. 1-546.

Ahmed, Z.U., Z.N.T. Begum, M.A., Hassan, M. Khondker, S.M.H. Kabir, M. Ahmad, A.T.A. Ahmed, A.K.A. Rahman and E.U. Haque (Eds) 2009c. Encyclopedia of Flora and Fauna of Bangladesh Vol. 8. Angiosperms: Dicotyledons (Fabaceae - Lythraceae). Asiatic Society of Bangladesh, Dhaka, pp. 1-478.

Ahmed, Z.U., Z.N.T., Begum, M.A., Hassan, M., Khondker, S.M.H., Kabir, M., Ahmad, A.T.A., Ahmed, A.K.A. Rahman and E.U. Haque (Eds) 2009d. Encyclopedia of Flora and Fauna of Bangladesh Vol. 9. Angiosperms: Dicotyledons (Magnoliaceae - Punicaceae). Asiatic Society of Bangladesh, Dhaka, pp. 1-488.

Ahmed, Z.U., Z.N.T., Begum, M.A., Hassan, M., Khondker, S.M.H., Kabir, M., Ahmad, A.T.A., Ahmed, A.K.A. Rahman and E.U. Haque (Eds) 2009e. Encyclopedia of Flora and Fauna of Bangladesh, Vol. 10. Angiosperms: Dicotyledons (Ranunculaceae - Zygophyllaceae). Asiatic Society of Bangladesh, Dhaka, pp. 1-580.

Alexiades, M. N. (ed.). 1996. Selected Guidelines for Ethno botanical Research: A Field Manual. The New York Botanical Garden, New York.

Ara, H., B. Khan and S. N. Uddin. (eds.) 2013. Red data book of vascular plants of Bangladesh, Vol 2. Bangladesh National Herbarium, Dhaka, Bangladesh. 280 pp

Bangladesh Bureau of Statistics 2011. Monthly Statistical Bulletin, December 2011. Statistics Division, Ministry of Planning, Government of the People's Republic of Bangladesh.

Cronquist, A. 1981. An integrated system of classification of flowering plants. Columbia University Press, New York, pp. 1262

Hooker, J.D. 1872-1897. The flora of British India. Vol. 1-7, London.

Hyland, B.P.M. 1972. A technique for collecting botanical specimens in rain forest. Flora Malesiana Bulletin, 26: 2038-2040.

Khan, M.S., M.M. Rahman, A.M. Huq, M.M.K. Mia, and M. A. Hassan. 1994. Assesment of biodiversity of Teknaf game reserve in Bangladesh focusing on economically and ecologically important plants species. Bangladesh J. Plant. Taxon. 1(1): 21-33.

Khan, M.S. and A.M. Huq 2001. The vascular flora of Chunati wildlife sanctuary in south Chittagong, Bangladesh. Bangladesh J. Plant.Taxon. 8(1): 47-64.

Khan M.S., M.M. Rahman and M.M. Ali (eds.). 2001. Red Data Book of Vascular Plants of Bangladesh. Bangladesh National Herbarium. pp. 179

Khan, M.S., M.M. Rahman, A.M. Huq, M.M.K. Mia and M. A. Hassan. 1994. Assessment of biodiversity of Teknaf game reserve in Bangladesh focusing on economically and ecologically important plants species. Bangladesh J. Plant. Taxon. 1(1): 21-33.

Prain, D. 1903. Bengal Plant. First Indian Reprint 1963, Bishen Singh Mahendra Pal Singh Dehra Dun. Vol. 1-2: 1-1013 pp.

Rahman, M.A. and S.B. Uddin 1998. Some anti-rheumatic plants used by tribal people of Hill Tracts district. Biodiversity Newsletter, University of Chittagong. 2(2): 4.

Rahman, M.O. and M. Begum, M.W. Ullah. 2013. Angiosperm flora of Sadar upazila of Munshiganj District Bangladesh. Bangladesh J. Plant Taxon. 20(2): 213-231.

Rahman, M.O. and M.A. Hassan 1995. Angiospermic flora of Bhawal Narional Park, Gazipur, Bangladesh. Bangladesh J. Plant Taxon. 2(1\&2): 47-79

Rahman, M.O. and M.A. Hassan. 1995. Angiospermic flora of Bhawal Narional Park, Gazipur, Bangladesh. Bangladesh J. Plant Taxon. 2(1\&2): 47-79.

Sarker M. H. 2012. "Sreenagar Upazila" in Sirajul Islam and Ahmed A. Jamal, Banglapedia: National Encyclopedia of Bangladesh (Second ed), Asiatic society of Bangladesh. 
Siddiqui, K.U., M.A. Islam, Z.U. Ahmed, Z.N.T. Begum, M.A. Hassan, M. Khondker, M.M. Rahman, S.M.H. Kabir, M. Ahmad, A.T.A. Ahmed, A.K.A. Rahman and E.U. Haque (Eds) 2007c. Encyclopedia of Flora and Fauna of Bangladesh, Vol. 11. Angiosperms: Monocotyledons (Agavaceae -Najadaceae). Asiatic Society of Bangladesh, Dhaka, pp. 1399.

Uddin, M.Z, M.F. Alam, A.S.M. Rahman and M.A. Hassan. 2011. Plant Biodiversity of Fashiakhali Wildlife Santuary, Bangladesh. Accepted for publication in First Bangladesh Forestry Congress Proceeding.

Uddin, M.Z. and M.A. Hassan. 2004. Flora of Rema-Kalenga Wildlife Sanctuary. IUCN Bangladesh Country Office, Dhaka, Bangladesh, vi+120 pp.

Uddin, M.Z. and M.A. Hassan. 2010. Angiosperm diversity of Lawachara National Park (Bangladesh): a preliminary assessment. Bangladesh J. Plant Taxon. 17 (1): 9-22.

Uddin, S.B. and M.A. Rahman. 1999. Angiospermic flora of Himchari National Park, Cox's Bazar, Bangladesh. J. Plant Taxon. 6(1): 31-68

Uddin, M.Z., M. F. Alam, M. A. Rahman and M. A. Hassan. 2013. Diversity in angiosperm flora of Teknaf Wildlife Sanctuary, Bangladesh. Bangladesh J. Plant Taxon. 20(2): 145-162.

Uddin, S.N., M.S. Khan, M.A. Hassan and M.K. Alam 1998. An annotated checklist of angiospermic flora of Sitapahar at Kaptai in Bangladesh. Bangladesh J. Plant Taxon. 5(1): $13-46$

Uddin, M. Z. and M. A. Hassan. 2010. Angiosperm diversity of Lawachara National Park (Bangladesh): a preliminary assessment. Bangladesh J. Plant Taxon. 17(1): 9-22.

Uddin, M. Z. and Md. Abiabdullah. 2016. Taxonomic study on the angiosperms of char kukri mukri wildlife sanctuary, Bhola district. J. Asiat. Soc. Bangladesh, Sci. 42(2): 153-168. 